\title{
A Study on the Distinguish of the Defective Product of Ceramic Toilet by Sound Characteristics
}

\author{
Bong-Young Kim, Eun-Young Yi and Myung-Jin Bae \\ Department of Information and Telecommunication Engineering, University of Soong-sil, \\ Sangdo-ro, Dongjak-gu, 369 Seoul, Korea
}

\begin{abstract}
The most important function of the toilet is to drain the excrement without polluting the water supply. The invention of a very sanitary ceramic toilet has revolutionized health hygiene. Since, ceramic toilet was first introduced to Korea in 1958, it played an important role in developing public health hygiene. Ceramic toilets can cause deformation and cracks in the manufacturing process. The ceramic toilet is very hard and its internal structure is complicated. Due to the nature of the product, there is no proper inspection method to check the internal condition. The inspector identifies the failure of the ceramic toilet through percussion inspection. When a ceramic toilet is tapped with a wooden hammer, a sound is produced according to the characteristics of the product. In the case of a defective product, the sound differs from the sound of the normal product memorized by the inspector. In this study, we tried to check whether the badness can be distinguished by using sound of each state of urinal. The normal products resonated at $650 \mathrm{~Hz}$ and $1 \mathrm{kHz}$ with similar energy size while the defective products showed different energy levels between resonance points or resonance points themselves. In conclusion, the examiner memorizes the sound components of each part of the normal product and distinguishes the difference of the fine sound components that occur when defective products are hit by the hearing characteristic.
\end{abstract}

Key words: Toilet, ceramic toilet, sound component, percussion inspection, hearing characteristic, defective product

\section{INTRODUCTION}

The toilet is a facility for discharging feces. A water closet toilet made in ancient Babylonia was discovered more than 2500 years ago. The most important function of the toilet is to drain the excrement without polluting the water supply. The great writer of France, Victor Hugo, said, 'Human history is the history of the toilet'. The bathroom represents the hygiene concept of a civilization and is seen as a measure of civilization development. In the Roman era, a very hygienic toilet with excellent water supply and drainage was used. However, in medieval age, the toilet culture of the primitive agricultural lifestyle was returned and even the royal family used a very unsanitary toilet. This caused repeated epidemics such as cholera and pest. Hygienic non-sanitary toilets adversely affect public health and in the past it was also a major source of terrible infectious diseases. The very hygienic ceramic toilet used in modern times was first made by a British pottery in the 1870's and since, then ceramic toiletries have made a splendid development in the toilet industry. Modern ceramic toilets were introduced to Korea in 1958 when it was installed in Jongam apartment complex in Seoul. Ceramic toilets which were in great demand in Korea in the 1970 and $1980^{\prime} \mathrm{s}$, made the toilet come into the house from outside the house. It also played an important role in promoting public health hygiene (Fig. 1).

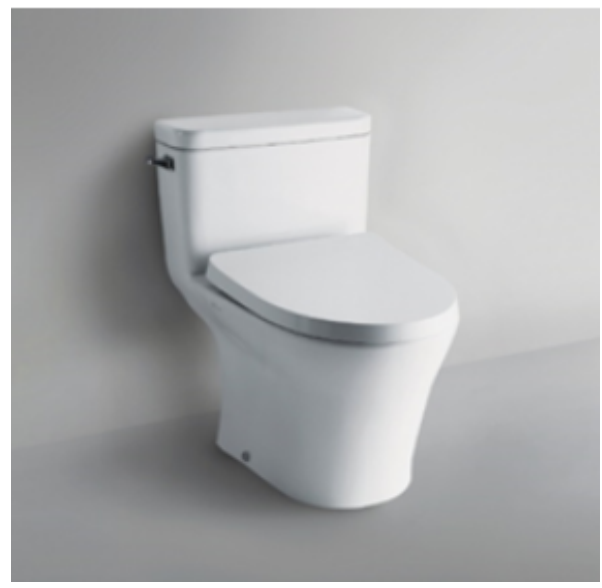

Fig 1: Ceramic toilet

Corresponding Author: Myung-Jin Bae, Department of Information and Telecommunication Engineering, University of Soong-sil, Sangdo-ro, Dongjak-gu, 369 Seoul, Korea 
If you try to knock a ceramic toilet with a wooden hammer, the ceramic toilet gives a clear iron sound. Ceramic is harder than steel and the harder it is the closer it is to an iron sound. The inspector identifies the failure of the ceramic toilet through percussion inspections that hear the sound of a wooden hammer and visual inspections that detect cracks of the product by the naked eye. If you hit the defective product, the ceramic toilet, it will appear somewhat different from the sound of the normal product because of the defective condition. The skilled inspectors identify the minor sound differences that can be heard when knocking on a ceramic toilet bowl and determine whether the product is defective very quickly. Percussion inspection of ceramic toilets makes it possible to conduct nondestructive inspection using human hearing characteristics (Bong-Young and Myung-Jin, 2017a, b; 2018; Lee et al., 2017; Sun-Young and Myung-Jin, 2015a, b; Kim and Bae, 2017; Bong-Young et al., 2017).

In this study, we have confirmed the sound characteristics of the ceramic toilet bowl when it is a normal product and when it is a defective product.

\section{MATERIALS AND METHODS}

\section{Basic theory for analyzing the sound components}

Resonance: When a force is applied from the outside of an object (or substance), if the natural frequency of the object (or substance) is equal to the frequency of the external force, the vibration becomes larger and the amplitude becomes larger. This phenomenon is called resonance. The natural frequency that causes resonance depends on the state of the object (or material). In the case of a solid, it has a natural frequency due to its shape, dimension, tension, elasticity, density, hardness, etc. In the case of liquid, it has a natural frequency depending on the dimension, elasticity, density, temperature and etc. The standard of the product is determined by the ceramic toilet. However, defective products that are out of specification due to cracks, etc. have different resonance characteristics from normal products due to differences in shape, elasticity, density and hardness (Bae and Lee, 1998).

Time domain analysis: The sound changes with the passage of time, depending on the type of energy change. Time domain analysis analyzes the speech signal by dividing it into short sections which are converted to parameter signals that change more slowly than the speech signal and analyze waveform changes over time. Through this analysis, the increase time, the decay time, the reverberation time and etc. can be known (Bae and Lee, 1998; Kang, 2007; Lee, 2004).

Frequency domain analysis: It can be said that all sounds are made up of various combinations of frequency components. The sound wave form used in the time domain is made up of a combination of several frequency components but by itself it is very difficult to know the state of the frequency-dependent combination. The frequency domain is an area whose frequency is independent variable for the signal. The sound signal can be represented by frequency domain information through Fast Fourier Transform (FFT). In this way, the frequency domain information can be analyzed for the size of each frequency and the combination of frequency components. In the frequency domain, the expression of the signal amplitude on the vertical axis with the horizontal axis as the frequency is called the frequency spectrum (Bae and Lee, 1998; Kang, 2007; Lee, 2004).

Manufacturing process and sound characteristics of ceramic toilet

Manufacturing process of ceramic toilet: Ceramic toilet is a hygienic facility that has to drain the faeces without polluting the water and to wash the dirt in the toilet cleanly with water to maintain its sanitary condition. Ceramic Toilet is manufactured in various forms depending on its use and type of water supply and drainage. Ceramic toilet is a kind of ceramics, it is put into a frame, dried, heated to high temperature, glazed and dried. The ceramic toilet is made of clay, so, it is converted to ceramics at very high temperature and the soil is completely melted and turned into ceramic component. The ceramic component made is harder than iron and becomes steel like. The hardness of the ceramic is very strong, so, if you tap it you will get an iron sound. Ceramic toilets are completed by inspectors through percussion inspection and visual inspection. Ceramic toilets can deform during the drying and heating of ceramics and if cracks occur, they become defective products.

Sound characteristics of ceramic toilet: The most commonly used non-destructive inspection method for ceramic toilet is percussion inspection. The percussion inspection is a method by which the inspector can tap the outer of the ceramic toilet with a stick to determine the 
deformation, crack and under specification of the product by sound or vibration. Because of the nature of the ceramic toilet, it is difficult to confirm the internal state of the product only by visual inspection and the percussion inspection is almost the only non-destructive inspection because of the limitation of quality measurement cost. Ceramic toilet is a pottery made from clay. When you hit it with a wooden hammer, you hear a clear sound like a metal iron. The shape of ceramic toilet is mostly blocked on one side and open on one side. This shape is similar to the shape of a species. Ceramics play the role of diaphragm and center space plays the role of soundbox. Since, ceramic toilet is not a simple closed container structure but a complex shaped frame, so, when you tap it makes a different sound for each part. In addition, since, the shape of each product differs according to use, different kinds of sounds are produced for different products. If you tap the same position for the same type of ceramic toilet, the same sound will be produced even if the other product is the same because the condition of the product is the same. However, even with the same type of ceramic toilet, the defective product has a complicated internal structure due to cracks and the like which affects the sound characteristics, resulting in a sound different from the normal product.

\section{RESULTS AND DISCUSSION}

To compare the sound of the ceramic toilets, we used the sound of a ceramic hammer with a normal state and defective state when tapped with a wooden hammer. For each sound source collected through the experiment, sound characteristics were analyzed through sound waveform and frequency spectrum. The collected sound source was sampled at $48 \mathrm{kHz}$ and quantized to 16 bits. The software used for the analysis was Audition CC and Cool Edit Pro 2.1.
Figure 2 compares and analyzes the sound waveforms of the sounds produced when the toilet seat which is a normal product is tapped for each part. Figure 3 shows the frequency spectrum transformed by the FFT for the sound wave form of Fig. 2. Figure 2 and 3 show that each part of the toilet seat has a different sound component and a different sound is emitted.

The sound wave form for the toilet seat in Fig. 2 shows a different sound pattern, depending on the position of the product. This is because the state of the object differs depending on the knocking position and the characteristics of the ringing appear differently.

Figure 3 shows that a high-frequency component of $900-4 \mathrm{kHz}$ is produced in all parts of the ceramic material, resulting in a cheerful iron sound. It also has different sound components depending on the shape of each part of the product. However, in the case of location 4 and 5 which are left and right symmetric points of the toilet seat, sound components are almost the same because they have the same structure based on the knocking point. That is if the structure is the same with respect to the knocking point, it can be seen that the same sound is produced by other products.

Figure 4 shows the frequency spectrum for each sound that appears when a normal product and a defective productl, a defective product 2 is tapped on a urinal product.

Figure 4 shows the frequency spectrum for each sound that appears when a normal product and a defective product 1 , a defective product 2 is tapped on a urinal product. Figure 4 shows the resonance frequency of urinal in each state. The normal state urinal resonates at the frequencies of $650 \mathrm{~Hz}$ and $1 \mathrm{kHz}$ and the second resonance point, $1 \mathrm{kHz}$ is $1 \mathrm{~dB}$ higher than the first

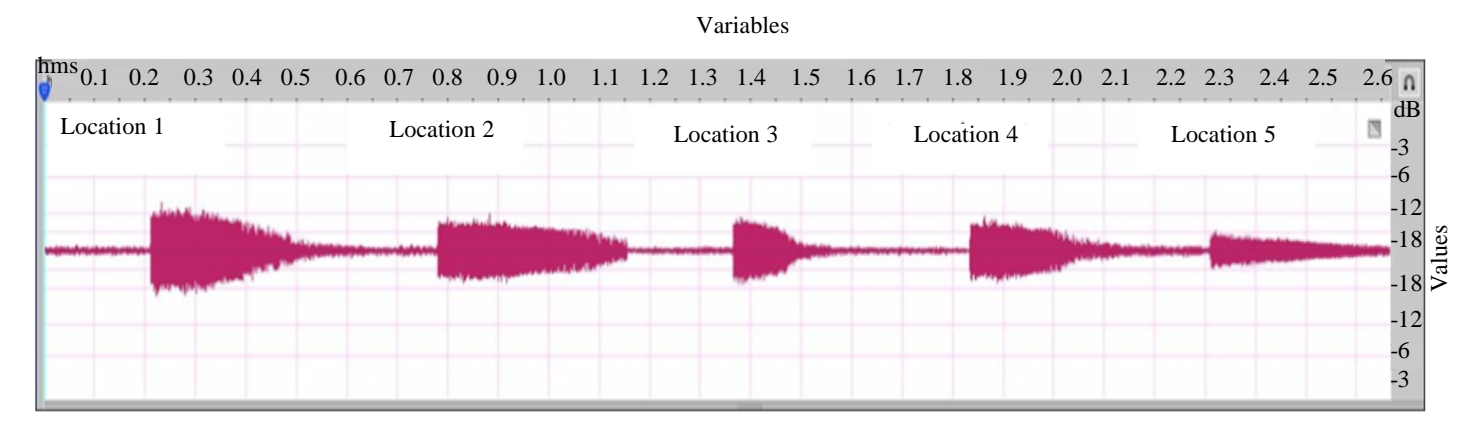

Fig. 2: Sound component supplied to the candlelight (wave form and frequency spectrum) 


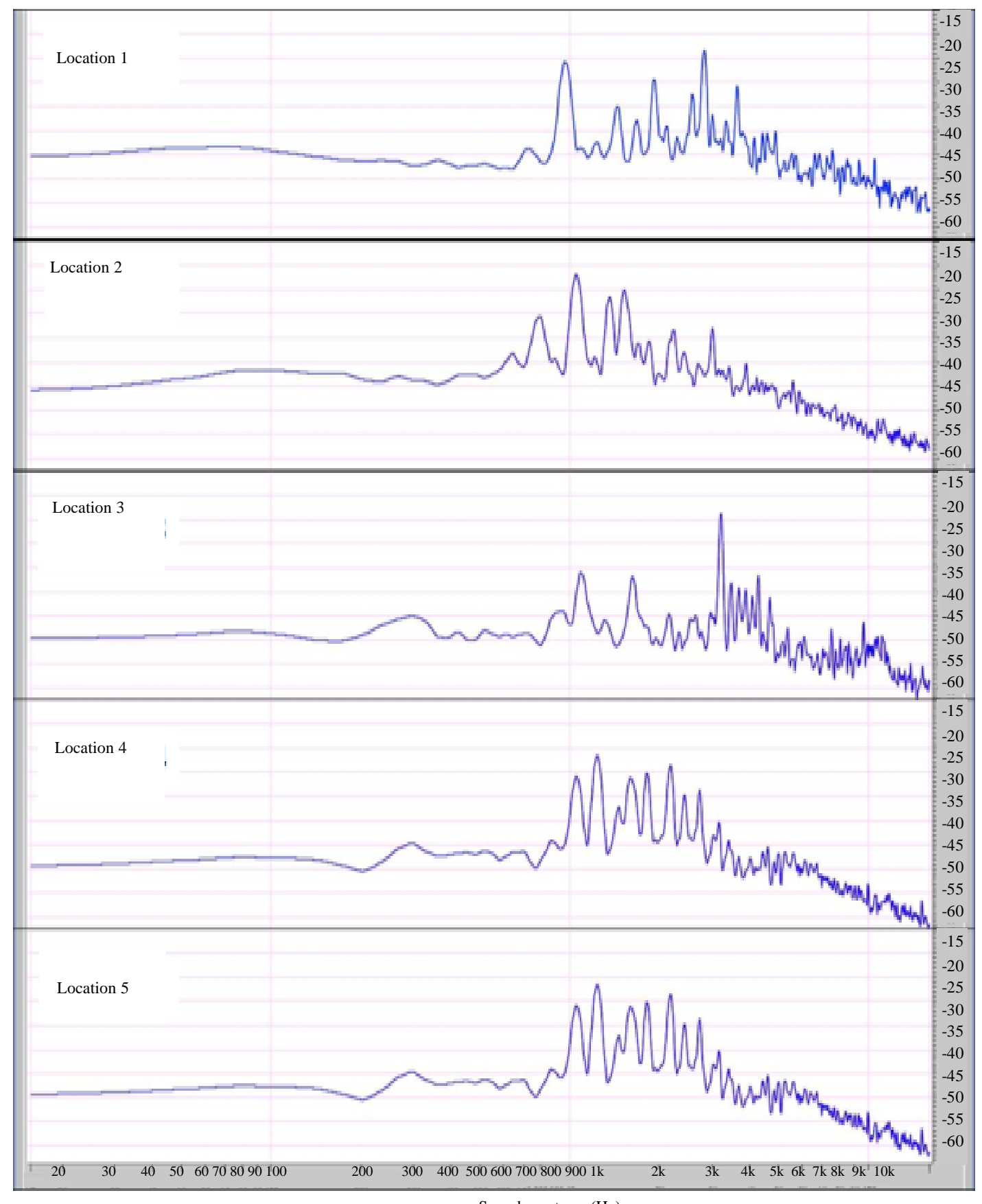

Sound spectrum $(\mathrm{Hz})$

Fig. 3: Sound spectrum for each part of toilet seat

resonance point, $650 \mathrm{~Hz}$. However, in the case of defective product 1 , it can be seen that the first resonance point $650 \mathrm{~Hz}$ is $3 \mathrm{~dB}$ higher than $1 \mathrm{kHz}$ which is the second resonance point, so that, it has about twice the sound power. In the case of defective product 2 , the first resonance point was $1 \mathrm{kHz}$ and the resonance point itself was different from the normal product. This can be said to be more strongly resonant at $650 \mathrm{~Hz}$ due to the state change of the product. In the case of defective product 2 , the resonance point itself is different from the normal product and the main resonance of $650 \mathrm{~Hz}$ and $1 \mathrm{kHz}$ disappears and the vibration is divided due to the product crack. 


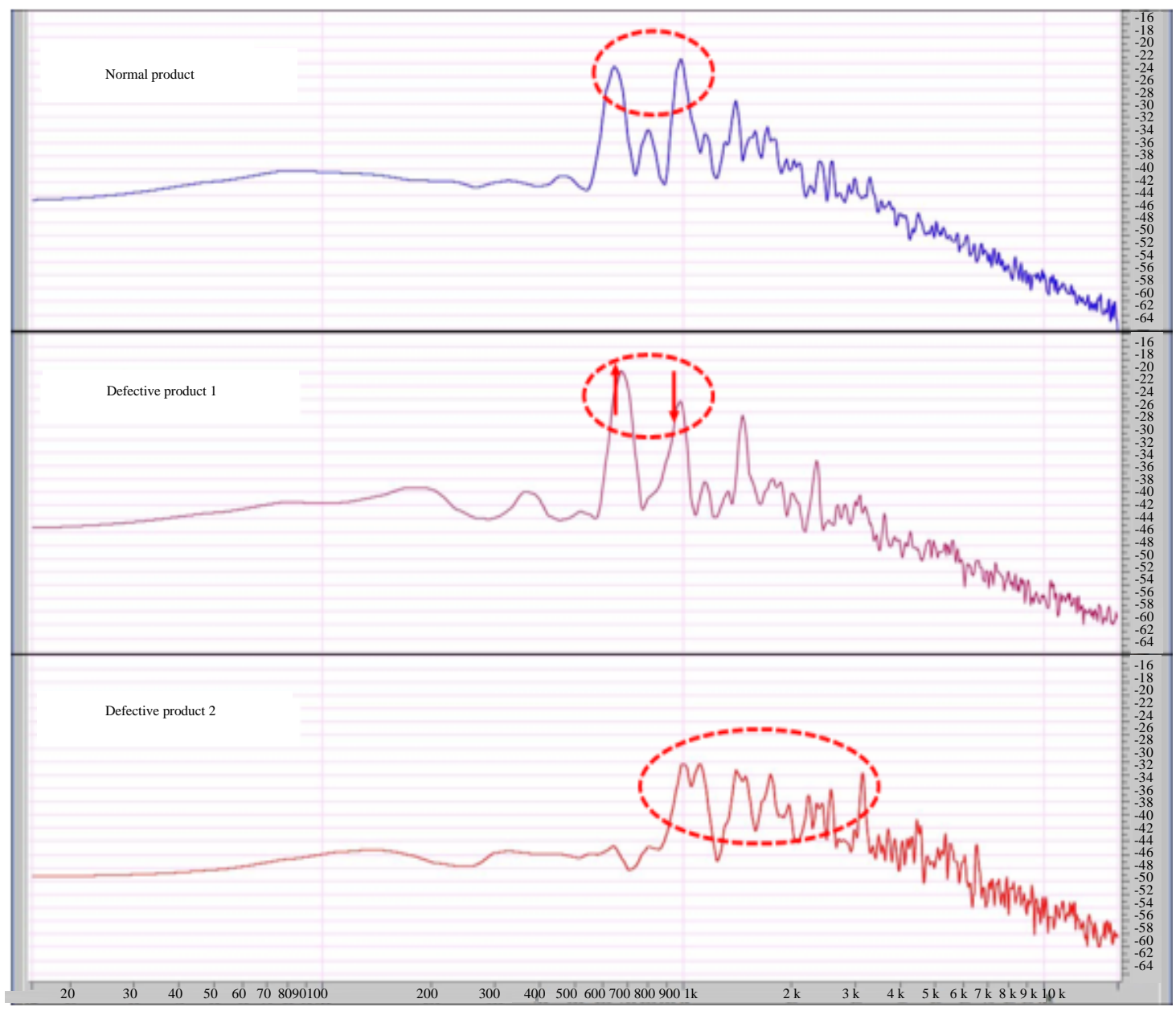

Sound spectrum (Hz)

Fig. 4: Spectrum of sound source by urinal condition

\section{CONCLUSION}

The most important function of the toilet is to drain the excrement without polluting the water supply. Hygienic non-sanitary toilets adversely affect public health and in the past it was also a major source of terrible infectious diseases. The invention of a very sanitary ceramic toilet has revolutionized health hygiene. The ceramic toilet is very hard due to the nature of the ceramic, so, it does not change its appearance until it is broken. And because of the high hardness, tapping a ceramic toilet bowl produces an iron sound. Ceramic toilets can crack and deform during drying and heating. Ceramic toilets are inevitably subjected to all inspections due to the nature of the products and non-destructive inspection is difficult. Therefore, it depends on the percussion inspection and visual inspections of inspectors. However, as percussion inspection depends entirely on the auditory ability of the examiner, the discrimination depends on the ability and condition of the examiner. Therefore, if we can distinguish the sound component of the ceramic toilet, it will be easier to identify the defective product. As a result of the experiment, we could confirm different sound characteristics according to the tapping position of the ceramic toilet. In the percussion experiment of urinal products, the normal product resonates at similar frequencies at $650 \mathrm{~Hz}$ and $1 \mathrm{kHz}$ but the defective product has a different resonance frequency or different energy magnitudes by resonance frequency. It is confirmed that this difference makes it possible to distinguish between normal product and defective product. When a ceramic toilet becomes a defective product due to cracks and deformation, the resonance itself changes, the resonance energy transfer characteristics change and the sound characteristics of the sound changes. This change changes the combination of the sound components, resulting in a fine but different sound. These results indicate that the ceramic toilet inspector memorizes the 
sound components of each part of the product and then identifies the differences in the fine sound components by the hearing characteristic when the defective product is tapped. Although, the experimental results of this study are only the sound characteristics of a specific product, it is possible to make systematic discrimination of defective products through the sound by setting the sound characteristics of the steady state of each part of the ceramic toilet. It is also a meaningful result that it is confirmed that non-destructive inspection is possible by processing the signal of sound for an object which is easily damaged or whose internal characteristics are unknown.

If the signal processing using the sound is limited to only a few parameters, it will be possible to perform the nondestructive inspection within a very short time. Through this study, it is expected that the sound signal processing can be utilized to be useful in the inspection field of the product.

\section{REFERENCES}

Bae, M.J. and S.H. Lee, 1998. Digital Speech Analysis. Dong Young Diamond Industrial Co. Ltd., South Korea.

Bong-Young, K. and B. Myung-Jin, 2017b. A study on sound characteristics according to canned goods status. Proc. Convergence Res. Lett. Convergent Art Humanities Sociology, 3: 1453-1456.

Bong-Young, K. and B. Myung-Jin, 2017a. A study on the sound investigation of drum-sound rock. Proc. Acoustical Soc. Korea Conf., 34: 1-17.
Bong-Young, K. and B. Myung-Jin, 2018. A study on the soundcharacteristics according to the pressure change of the airtight container. Proc. Convergence Res. Lett. Convergent Art Humanities Sociology, 4: 1889-1892.

Bong-Young, K., P. Hyung-Woo and B. Myung-Jin, 2017. Study of similarity between stone bell sound in maneo temple and normal bell sound. Asia Pac. J. Multimedia Serv. Convergent Art Humanities Sociology, 7: 881-888.

Kang, S.H., 2007. Fundamentals of sound engineering. Sound Media, Korea.

Kim, B.Y. and M. Bae, 2017. A study on the sound investigation of wooden gong rock. J. Acoustical Soc. Am., 142: 2606-2606.

Lee, S.T., 2004. Principles and Application of Sound. Cheong Moon Gak, Korea,

Lee, W.H., B.M. Lim and M.J. Bae, 2017. A study on the identification of watermelon using acoustic characteristics. Asia Pac. J. Multimedia Serv. Convergent Art Humanities Sociology, 7: 271-278.

Sun-Young, P. and B. Myung-Jin, 2015b. Analysis on rolling sound of normal and faulty Ruditapes philippinarum. Asia Pac. J. Multimedia Serv. Convergent Art Humanities Sociology, 5: 593600 .

Sun-Young, P. and B. Myung-Jin, 2015a. Analysis on rolling sound of normal and faulty short-necked clams. Proc. Convergence Res. Lett. Convergent Art Humanities Sociology, 1: 111-115. 